\title{
BROADBAND JAMMER EXCISION IN SPREAD SPECTRUM COMMUNICATION SYSTEMS USING TIME-FREQUENCY MASKING
}

\author{
Aydın AKAN and Ertuğrul SAATÇI \\ University of Istanbul, Department of Electrical and Electronics Engineering \\ Avcilar 34850, Istanbul TURKEY \\ e-mail: akan@istanbul.edu.tr
}

\begin{abstract}
In this paper we demonstrate the use of the evolutionary spectral masking based on a multi window Gabor expansion in excising broadband jammers in spread spectrum communication systems. In this method the jammer signals can be localized in the time-frequency plane and then be removed without severely distorting the original signal. Simulation results show that this technique improves the bit error performance of the receiver compared to the case where there is no excision. Experimental results illustrating the performance of our algorithm are presented.
\end{abstract}

Key-Words: Spread Spectrum Communication, Jammer Excision, Time-Frequency Analysis, Discrete Gabor Expansion, Time-Frequency Masking.

\section{ÖZET}

Bu çalışmada yayıl izge iletişim sistemlerindeki geniş bandlı bozucu girişlerin evrimsel maskeleme kullanılarak temizleme problemi incelenmektedir. Bu yöntem ile bozucu giriş sinyalleri zaman sıklk düzleminde yerelleştirilerek, mesaj sinyaline fazla zarar vermeden giderilebilirler. Benzetim sonuçları bu tekniğin alıcıdaki bit hata oranını iyileştirdiğini göstermiştir. Önerilen bu algoritmanın başarımı örnekler ile sunulmaktadır.

Anahtar Kelimeler: Yayıli İze İletişim, Bozucu Giriş Giderme, Zaman Sıklık Analizi, Ayrık Gabor Açılımı, Zaman Siklık Maskelemesi.

\section{INTRODUCTION}

Spread Spectrum (SS) communication systems are widely used in transmission environments where there are high power jamming interferences or multipath problems [1]. The main characteristic of SS systems is that the transmitted signal occupies a bandwidth much wider than the bandwidth necessary to send the information. Spreading of the information signal spectrum is accomplished by modulating it with a spreading or code signal before transmission [1]. This spreading of the transmitted signal energy over a wide frequency band allows the SS communication system to become immune to outside interferences or jammers during transmission. The most widely used spreading signal is the direct sequence (DS) or pseudorandom noise (PN) sequence.

Authors are with the Department of Electrical and Electronics Engineering, University of Istanbul, Avcilar 34850, Istanbul TURKEY. Tel. +90(212) 694-8103, Fax. +90(212) 591-1997, Email: akan@istanbul.edu.tr. This work was supported by The Research Fund of The University of Istanbul, Project numbers: ÖIII/67/010699 and Ö-III/77/11102000
The information sequence is modulated by this higher rate PN sequence at the transmitter end, spreading the spectrum of the signal. At the receiver end, the received signal is demodulated by using the synchronized replica of the PN sequence to recover the information signal. Meanwhile the energy level of narrowband jammers added to signal during transmission is reduced by spreading it accross the bandwidth of the PN sequence. Therefore, direct sequence spread spectrum (DSSS) systems are inherently immune to narrowband interferences. However, when the jamming signal is broad-band, which destroys too many information bits, or signal-to-noise ratio (SNR) is too low, error performance of SS systems dramatically degrades. The performance of the SS communication systems can be improved in terms of immunity to interference or jammers by estimating interference and subtracting it from the received signal prior to despreading and demodulation. Removing the jammer in SS communication systems is an important issue especially in military applications. This filtering operation suppresses the interference level and increases the SNR [2], 
Furthermore, by substituting for the Gabor coefficients in the above equation, we have that

$$
A(n, k)=\sum_{\ell=0}^{N-1} x(\ell) \mathrm{w}(n, \ell) e^{-j \ell \frac{2 \pi}{K} k}
$$

with the window defined as

$$
\mathrm{w}(n, \ell)=\frac{1}{I} \sum_{i=0}^{I-1} \sum_{m=0}^{M-1} \gamma_{i}^{*}(\ell-m L) h_{i}(n-m L) .
$$

Equation (6) can be viewed as a short-time Fourier transform with a time-varying window. The ES calculation can then be done either by using (5) or by averaging $A_{i}(n, k)$, obtained from different scales, using different averaging techniques [7]. Then the evolutionary spectrum of $x(n)$ is obtained according to

$$
S_{E S}(n, k)=\frac{1}{K}|A(n, k)|^{2}
$$

where the factor $1 / K$ is used for proper energy normalization. It is shown in [7] that normalizing the window $\mathrm{w}(n, \ell)$ to unit energy, the total energy of the signal is preserved. Furthermore, $S_{E S}(n, k)$ is always non-negative and approximates the marginal conditions [18]. Hence, in contrary to many other time-frequency distributions, it is easily interpretable as joint energy density function [7]. In the next section, we employ the above evolutionary spectral estimate, for the time-frequency jammer excision in spread spectrum communication systems.

\section{JAMMER EXCISION USING TIME- FRE- QUENCY MASKING}

We develop an evolutionary spectral masking for removing jammers in SS communication signals. Masking in the time-frequency domain involves modifying the evolutionary kernel by a TF mask function. In time-frequency, there are two ways to change the energy distribution of a signal: filtering and masking [19], [20], [21], [22], [23]. Timefrequency distributions (TFDs), especially the Wigner distribution, have been applied to the time-frequency masking and signal synthesis [24], [25], [26], [27], [28]. However, the modified TFD is not necessarily valid since it may not correspond to any signal. For instance, time-frequency masking using the Wigner distribution often requires approximation using a least square minimization [21], [28]. Cumbersome approximation algorithms makes time-frequency masking and signal synthesis with the Wigner distribution thus rather undesirable.

In the evolutionary case, the masking is achieved by multiplying $A(n, k)$ by a masking function $G(n, k)$ to attain the representation of the masked signal. We will show that by performing the masking in the Gabor space, only the support of the Gabor coefficients is changed by the masking and thus the masked signal can be easily approximated from the original representation.
The objective is to pass the signal components located inside the mask but suppress all components located outside. If the mask is given as

$$
G(n, k)= \begin{cases}1 & (n, \omega) \in R \\ 0 & \text { otherwise }\end{cases}
$$

where $R$ denotes the pass region of the mask, and the representation of the input signal is as before

$$
\begin{aligned}
x(n) & =\sum_{k=0}^{K-1} A_{x}(n, k) e^{j n \frac{2 \pi}{K} k} \\
& =\frac{1}{I} \sum_{i=0}^{I-1} \sum_{m=0}^{N-1} \sum_{k=0}^{K-1} a_{i, m, k} h_{i}(n-m L) e^{j n \frac{2 \pi}{K} k}(9)
\end{aligned}
$$

then the masked signal $y(n)$ can be expressed by

$$
y(n)=\sum_{k=0}^{K-1} A_{y}(n, k) e^{j n \frac{2 \pi}{K} k}
$$

where

$$
A_{y}(n, k)=A_{x}(n, k) G(n, k) .
$$

Consider then an approximate implementation of the mask function, i.e.,

$$
G(n, k) \approx \sum_{j=0}^{J-1} t_{j}(n) f_{j}(k)
$$

where $t_{j}(n)$ and $f_{j}(k)$ are the time and frequency windows, respectively, defined as

$$
t_{j}(n)= \begin{cases}1 & N j / J \leq n<N(j+1) / J \\ 0 & \text { otherwise }\end{cases}
$$

and

$$
f_{j}(k)= \begin{cases}1 & k_{1 j} \leq k \leq k_{2 j} \\ 0 & \text { otherwise }\end{cases}
$$

for $j=0, \cdots, J-1$. Replacing equations (11) and (12) in (10), $y(n)$ is approximately synthesized by

$$
\begin{aligned}
y(n) \approx \sum_{j=0}^{J-1} y_{j}(n) & =\sum_{j=0}^{J-1} \sum_{k=0}^{K-1} A_{x}(n, k) e^{j n \frac{2 \pi}{K} k} t_{j}(n) f_{j}(k) \\
& =\sum_{j=0}^{J-1} \sum_{k=k_{1 j}}^{k_{2 j}} A_{x}(n, k) e^{j n \frac{2 \pi}{K} k} t_{j}(n)
\end{aligned}
$$

Notice that the effect of $f_{j}(k)$ is to change the frequency support of $A_{x}(n, k)$ and the time window $t_{j}(n)$ has the effect of limiting the support in time of $y_{j}(n)$.

Substituting for $A_{x}(n, k)$ from (5), we have

$$
y(n) \approx \sum_{j=0}^{J-1} \frac{1}{I} \sum_{i=0}^{I-1} \sum_{m=0}^{M-1} \sum_{k=k_{1 j}}^{k_{2 j}} a_{i, m, k} h_{i}(n-m L) e^{j n \frac{2 \pi}{K} k} t_{j}(n)
$$


Notice that the above is the original representation of $x(n)$ with the frequency limited according to the frequency band for each frame. The time window constrains the time support in each frequency window. The approximation of the frame can be improved by reducing the support of the time windows, at the cost of increased computation.

Thus our masking procedure consists in keeping the Gabor coefficients of the input signal in the desired frequency range $\left[k_{1 j}, k_{2 j}\right]$ and windowing in time. Reducing the size of the time windows or overlapping them, one can improve the results but at a higher computational cost.

The jammer excision by using the above masking procedure is achieved by multiplying the evolutionary kernel of the received, distorted signal $r(n)$ by a TF mask. The masking matrix $G(n, k)$ is generated by the following procedure: A set of spread spectrum information signals distorted by white noise are analyzed by using the proposed multi-window ES estimation method. Let the maximum value of these ES estimates be $\mu$. The entry $G(n, k)$ is set to zero for an $(n, k)$ TF point if the value of $A_{x}(n, k)$ is higher than $\mu$. Otherwise $G(n, k)$ is set to one, i.e.,

$$
G(n, k)= \begin{cases}0 & A_{x}(n, k)>\mu \\ 1 & \text { otherwise }\end{cases}
$$

The output after masking the jammer can then be synthesized through equation (14).

In the following, we show that this method can be effectively applied to removing narrow- and broadband jammers in high Jammer-to-Signal-Ratio (JSR) and low SNR environments by means of examples.

\section{EXPERIMENTAL RESULTS}

Example 1. In this example, a 16 bit long data sequence is multiplied by 1:16 PN sequence to spread the spectrum of the data. As corrupting white noise with

$\mathrm{SNR}=10 \log \left(\sigma_{s}^{2} / \sigma_{w}^{2}\right)=2 \mathrm{~dB}$, where $\sigma_{s}^{2}$ is the average power of the message signal, and $\sigma_{w}^{2}$ is the average power of the additive white noise is added to spreaded signal. Moreover, a linear chirp type jammer with JSR $=10 \log \left(\sigma_{j}^{2} / \sigma_{s}^{2}\right)=18 \mathrm{~dB}$, where $\sigma_{j}^{2}$ denotes the average power of the jammer, is added to the signal. In Fig. 2, ES of the spreaded signal is given. Fig. 3 shows the ES of the distorted signal. Without any preprocessing, the demodulated output signal showed 6 bit errors. Linear chirp jammer is excised by the propose method, and the ES of masked signal is given in Fig. 4. Recovered signal (solid line) is given together with the distorted signal (dashed line) in Fig 5. Bit error rate (BER) is calculated to be 0 out of 16 bits. It is shown from the figures that chirp jammer can be excised in the TF plane without loosing the information signal. To compare our result with some other methods, DSSS data is preprocessed using FFT based filtering ( 3 bit errors) and FRFT based excision [17] (2 bit errors). Bit error performance of our method is compared with that of other methods in Table 1 . We see from the table that the proposed TF masking method has the lowest bit error rate, hence the best bit error performance. 